\title{
Editorial
}

\section{Return to sport after injuries: no answer to the main question}

One of the questions most frequently put to a sports physician or orthopedist when an athlete sustains an injury is "when will he be able to play/compete again?". It is, I would say, the first question that everyone asks in this situation, and I refer to the athlete himself, as well as the hierarchy of people with an "interest" in his health (his trainer, club and agent), his family, and, in cases with high media impact, sports journalists and fans. It seems an obvious question, clear and straight forward, and from the perspective of the uninitiated, there is no reason why it should not have a clear and straight forward answer. However, the reply that is often given is deceptively simple: "we will have to wait and see, he will be back when he is ready". This reply actually conceals a rather complex problem in sports traumatology, namely the lack of an effective and objective instrument capable of providing a definite indication of the time it will take for an athlete to recover completely and be fit to return to his specific sport, in conditions that allow him to guarantee the same level of performance as before, and without the risk of re-injury.

In this issue of Joints, Grassi et al. provide an update on the grading of muscle injuries, which are recognized to be among the most frequent injuries occurring in the athletic population, accounting for more than 30\% of all injuries in professional soccer players (1). The Authors show that new comprehensive grading systems, such as the Munich Muscle Injury Classification, the ISMuLT classification and the British Athletic Classification, combining detailed imaging features with clinical presentation, are valid tools for assessing injury severity, and valid outcome measures for assessing the efficacy of treatments. However, since no functional evaluation is included in these systems, they are unable to answer to the main question: when will the athlete be ready to return to sport?

Although re-injury rate has been used in several clinical studies as a parameter for assessing the efficacy of sports injury treatments, and also as valid measure for assessing the prognostic value of a grading system, in my opinion, it amounts to little more than a post hoc evaluation of the validity of our original prediction regarding the timing of the patient's return to sport (i.e., we felt that the athlete was ready to return to sport, and it turned out that he wasn't).

Zanon et al. presented a study on the efficacy of platelet-rich plasma in the treatment of acute hamstring injuries in professional football players (2). Ultrasound-based and magnetic resonance-based classifications were used to assess the severity of muscle injury and to evaluate the healing process; from a clinical standpoint, patients were considered healed when they were once again available to play, and sport participation absence, measured in days, was taken to correspond to the time necessary for healing (3), which, once again, corresponds to the time necessary for the patient to reach the point "when he is ready".

There is no doubt that assessment of the physical conditions of an injured athlete must be based on his performance of a series of motor actions, which are used for establishing whether an individual is really fit to start practicing a given sporting activity again. Since many of the assessments used to this end - both clinical (e.g. tests of strength or agility) and instrumental (e.g. isokinetic tests or analysis of movement) - are based on a single test or on a combination of several individual tests, their general limitation is that they are unable to provide an overview. Recently, the use of objective generic evaluation forms able to detect functional deficits that may represent a potential risk of injury or re-injury has become widespread. Of these, the Functional Movement Screen (FMS) is currently the most widely used by leading professional athletes' associations (4). The FMS comprises seven exercises that assess flexibility, stability, strength, balance and coordination, seeking to identify any restriction or asymmetries. Each exercise is assigned a score of between 0 and 3. Recent studies have demonstrated a strong association between the risk of injury and an FMS score <14 (5). However, inter-observer reproducibility studies have shown very variable results for each exercise, with reproducibility coefficients ranging from 1 (perfect reproducibility) to 0.39 (poor reproducibility) (6).

Another important aspect is that an injured athlete does not want to return to sport per se, but rather to his specific sport, preferably practicing it at the same level as prior to the injury. Therefore, the functional assessment of an athlete should be linked not only to the disease, but also to the type of sport practiced. In this issue, Spennacchio et al. review the literature on outcome evaluation systems used in another traumatic condition that is highly prevalent in athletes: Achilles tendon rupture (7). Even though the Authors demonstrate that there exist numerous condition-specific subjective and objective assessment tools, only a few of these, such as The Foot and Ankle Ability Measure (8), contain items specifically designed for evaluating sports performance, and none of them is capable of assessing sport-specific functional abilities, as they fail to include anything more detailed than brief, general items such as: "ability to perform activity with your normal technique" or "ability to participate in your desired sport as long as you would like".

In other words, the responsibility for answering the main question lies with the individual directly involved: "You say you feel ready to return to sport? That means you are ready".

\section{References}

1. Grassi A, et al. An update on the grading of muscle injuries: a narrative review from clinical to comprehensive systems. Joints. 2016;4 (1):39-46.

2. Zanon G, et al. Platelet-rich plasma in the treatment of acute hamstring injuries in professional football players. Joints. 2016:4(1):17:23.

3. Balius R, Maestro A, Pedret C, et al. Central aponeurosis tears of the rectus femoris: practical sonographic prognosis. Br J Sports Med. 2009;43:818-824.

4. Cook G, Burton L, Hoogenboom B. Pre-participation screening: the use of fundamental movements as an assessment of function - part 1. N Am J Sports Phys Ther. 2006;1:62-72.

5. Kiesel K, Plisky PJ, Voight ML. Can serious injury in professional football be predicted by a preseason functional movement screen? N Am J Sports Phys Ther. 2007;2:147-158.

6. Shultz R, Anderson SC, Matheson GO, et al. Test-retest and interrater reliability of the functional movement screen. J Athl Train. 2013;48:331-336.

7. Spennacchio $P$, et al. Outcome evaluation after Achilles tendon ruptures. A review of the literature. Joints. 2016;4 (1):51-59.

8. Martin RL, Irrgang JJ, Burdett RG, et al. Evidence of validity for the Foot and Ankle Ability Measure (FAAM). Foot Ankle Int. 2005;26:968-983. 